\title{
4
}

\section{Classical and Quantum Semigroups}

Several quantum Markov semigroups admit an invariant abelian subalgebra which can be looked at as an algebra of bounded functions on some measurable space. The restriction to this subalgebra is a semigroup of positive and identity preserving operators i.e. a classical Markov semigroup (see Examples 3.3, 3.4).

We first study the following problem: given a classical Markov semigroup is it the restriction to an abelian subalgebra of a quantum Markov semigroup? The answer to this question would be a step towards the understanding which classical processes can appear in quantum stochastics. Here shall see that the answer is in the affirmative in for homogeneous diffusions on $\mathbb{R}^{d}$ with smooth coefficients (see also the partial result of [41]).

It can be shown that the answer is in the affirmative also for:

- countable state Markov chains (see [70]), [44]),

- some diffusion with non-smooth drift (e.g. the transient Bessel processes $[27])$.

- the Azéma martingales for parameters smaller than a critical value (see

A complete characterisation of classical semigroups which can arise as restrictions of quantum Markov semigroups however is not known. We point out the analytical difficulties related to the "quantum" interpretation of classical boundary conditions.

This problem, however, is also interesting from a probabilistic point of view because it is a natural generalisation of the theory of classical stochastic processes. In fact B.V.R. Bhat and K.R. Parthasarathy showed in [20] that every quantum Markov semigroup admits a canonical dilation to a quantum Markov process (in the appropriated sense).

In the second part of this chapter we apply our results to construct a class of quantum Markov semigroup arising in Quantum Optics in the weak coupling or singular coupling limit of a multi-mode Boson field interacting with a reservoir in 
the so-called "squeezed vacuum". These semigroups appear in several contexts in physical applications (see e.g. [10], [15], [47]).

\subsection{Preliminary definitions and results}

Let $E$ be a closed subset of $\mathbb{R}^{d}$ and let $\mathcal{E}$ be the Borel $\sigma$-field of $E$. Let $L^{\infty}(E ; \mathbb{C})$ (resp. $C_{l}^{0}(E ; \mathbb{C})$ ) be the Banach space of complex-valued measurable (resp. continuous) functions on $E$ (resp. having a limit as $|x| \rightarrow \infty$ if $E$ is unbounded) endowed with the norm

$$
\|f\|_{\infty}=\sup _{x \in E}|f(x)| .
$$

Moreover, for all integer $k \geq 1$, let $C_{l}^{k}\left(E ; \mathbb{C}^{\prime}\right)$ be the vector space of complexvalued functions on $E$ with all the partial derivatives of the first $k$ orders in $C_{l}^{0}(E ; \widetilde{C})$. Let

$$
P:[0,+\infty) \times E \times \mathcal{E} \rightarrow[0,1]
$$

be a transition probability. Consider the identity preserving semigroup $T=$ $\left(T_{t}\right)_{t \geq 0}$ on $L^{\infty}\left(E ; C^{\prime}\right)$ defined by

$$
T_{t} f(x)=\int_{E} f(y) P(t, x ; d y)
$$

Suppose that the semigroup $T$ enjoys the Feller property i.e.

1. for all $f \in C_{l}^{0}(E ; \mathbb{C})$ and all $t \geq 0$ the function $T_{t} f$ belongs to $C_{l}^{0}(E ; \mathcal{C})$,

2. the restriction of $T$ to $C_{l}^{0}(E ; \mathscr{C})$ is a strongly continuous contraction semigroup.

Clearly, in this case, the semigroup $T$ is uniquely determined by its restriction to $C_{l}^{0}(E ; \mathbb{C})$. Therefore we shall work with this restriction. Denote by $A$ the infinitesimal generator, the operator on $C_{l}^{0}(E ; \mathbb{C})$ defined by

$$
\begin{aligned}
D(A)=\left\{f \in C_{l}^{0}(E ; \mathbb{C})\right. & \left.\mid \lim _{t \rightarrow 0^{+}}\left(T_{t} f-f\right) / t \quad \text { exists in norm }\right\}, \\
A f & =\lim _{t \rightarrow 0^{+}}\left(T_{t} f-f\right) / t .
\end{aligned}
$$

Let $h=L^{2}(E ; \mathcal{C})$ and let $\mathcal{B}(h)$ be the algebra of all bounded operators on $h$. Clearly we have the embeddings

$$
\begin{aligned}
C_{l}^{0}(E ; \mathscr{C}) & \hookrightarrow & L^{\infty}(E ; \mathbb{C}) & \hookrightarrow \mathcal{B}(h) \\
f & \rightarrow & f & \rightarrow M(f)
\end{aligned}
$$

where $M(f)$ denotes the multiplication operator by $f$.

In order to extend the semigroup $T$ to a quantum Markov semigroup on $\mathcal{B}(h)$ as a first step we find a suitable representation for the infinitesimal generator $A$ allowing to find the operators $G$ and $L_{\ell}$ related to forms $£(M(f))$ for a multiplication operator $M(f)$ by a function $f$. 
Definition 4.1 We say that the infinitesimal generator $A$ can be represented in Lindblad form if there exists operators $G,\left(L_{\ell}\right)_{\ell=1}^{\infty}$ on $h$ satisfying the hypothesis AA such that

$$
\langle v,(A f) u\rangle=\langle v, f G u\rangle+\sum_{\ell=1}^{\infty}\left\langle L_{\ell} v, f L_{\ell} u\right\rangle+\langle G v, f u\rangle
$$

for all $v, u \in D(G)$ and all $f \in D(A)$.

The following proposition gives necessary conditions for an infinitesimal generator $A$ to be representable in Lindblad form.

Proposition 4.2 Suppose that $A$ can be represented in Lindblad form. Let 11. $: \in D(G)$ and let $f \in D(A)$.

1. We have the inequality

$$
|\langle v,(A f) u\rangle| \leq(\|u\|+\|v\|)(\|G u\|+\|G v\|)\|f\|_{\infty} .
$$

2. The non-negative function

$$
g=|v(G u)|+|u(G v)|+\sum_{\ell=1}^{\infty}\left|\left(L_{\ell} v\right)\left(L_{\ell} u\right)\right|
$$

belongs to $L^{1}(E ; \mathbb{C})$ and we have the inequality

$$
|\langle v,(A f) u\rangle| \leq \int_{E} g(x)|f(x)| d x .
$$

Proof. By Definition 4.1 and the Schwarz inequality we can estimate the modulus of the scalar product $\langle v,(A f) u\rangle$ by

$$
\begin{aligned}
& \left(\|G v\| \cdot\|u\|+\sum_{j=1}^{\infty}\left\|L_{j} v\right\|\left\|L_{j} u\right\|+\|v\| \cdot\|G u\|\right)\|f\|_{\infty} \\
\leq & \left(\|G v\| \cdot\|u\|+\frac{1}{2} \sum_{j=1}^{\infty}\left(\left\|L_{j} v\right\|^{2}+\left\|L_{j} u\right\|^{2}\right)+\|v\| \cdot\|G u\|\right)\|f\|_{\infty}
\end{aligned}
$$

Using the hypothesis AA we obtain the inequalities

$$
\begin{aligned}
& \sum_{j=1}^{\infty}\left\|L_{j} u\right\|^{2}=-2 \Re e\langle u, G u\rangle \leq 2\|u\|\|G u\|, \\
& \sum_{j=1}^{\infty}\left\|L_{j} v\right\|^{2}=-2 \Re \operatorname{Re}\langle v, G v\rangle \leq 2\|v\|\|G v\| .
\end{aligned}
$$

Then a simple computation yields (4.1). 
By the Schwarz inequality and hypothesis AA, for all $u, v \in D(G)$, we have

$$
\begin{aligned}
\int_{0}^{\infty} \sum_{j=1}^{\infty}\left|\left(L_{j} v\right)(x)\left(L_{j} u\right)(x)\right| d x & =\sum_{j=1}^{\infty} \int_{0}^{\infty}\left|\left(L_{j} v\right)(x)\right| \cdot\left|\left(L_{j} u\right)(x)\right| d x \\
& \leq \sum_{j=1}^{\infty}\left\|L_{j} v\right\|\left\|L_{j} u\right\| \\
& \leq-\Re e\langle v, G v\rangle-\Re e\langle u, G u\rangle \\
& \leq\|u\| \cdot\|G u\|+\|v\| \cdot\|G v\| .
\end{aligned}
$$

This shows that the function $g$ given by $(4.2)$ belongs to $L^{1}(E ; \mathbb{C})$. Therefore it is easy to obtain the inequality (4.3).

When $A$ can be represented in Lindblad form we can construct the minimal quantum dynamical semigroup on $\mathcal{B}(h)$ associated with $A$.

The following result can be applied to check whether it is an extension of the corresponding classical Markov semigroup generated by $A$.

Theorem 4.3 Suppose that $A$ can be represented in Lindblad form through operators $G, L_{\ell}$ and let $\mathcal{T}$ be the minimal quantum dynamical semigroup solving (3.15) with the given operators $G, L_{\ell}$. Then $\mathcal{T}$ is an extension of the classical semigroup $T$ generated by $A$ if and only if it is Markov.

Proof. If $\mathcal{T}$ is an extension of $T$ then, denoting by 1 the constant function equal to 1 on $E$, and identifying bounded functions with the corresponding multiplication operators, we have

$$
\mathcal{T}_{t}(\mathbb{1})=T_{t}(1)=1=\mathbb{1} .
$$

Therefore $\mathcal{T}$ is Markov.

Conversely, notice that, for every $f \in D(A)$, and $v, u \in D(G)$ we have

$$
\begin{aligned}
\left\langle v,\left(T_{t} f\right) u\right\rangle & =\langle v, f u\rangle+\int_{0}^{t}\left\langle v,\left(A\left(T_{s} f\right)\right) u\right\rangle d s \\
& =\langle v, f u\rangle+\int_{0}^{t}\left\langle v, £\left(T_{s} f\right) u\right\rangle d s
\end{aligned}
$$

Hence, if $\mathcal{T}$ is identity preserving, then by Corollary 3.23 we have

$$
\mathcal{T}_{t}(M(f))=M\left(T_{t}(f)\right)
$$

for every $t \geq 0$. The above identity yields also for $f \in C_{l}^{0}(E ; \mathbb{C})$ because the domain $D(A)$ is dense for the uniform norm.

This completes the proof.

Remark. Proposition 4.2 and an analogue of Theorem 4.3 also hold when the semigroup $T$ is $\mathrm{w}^{*}$-continuous, i.e. continuous with respect to the topology 
$\sigma\left(L^{\infty}, L^{1}\right)$. Here we deal with classical Markov semigroups enjoying the Feller property because they are widely studied in the literature.

The above discussion suggests to study quantum extensions of classical Markov semigroups in two steps:

1. represent the infinitesimal generator of the classical semigroup in Lindblad form,

2. show that the minimal quantum dynamical semigroup arising is identity preserving.

The analytical tools for the second step were already discussed.

The following heuristic remarks suggest a recipe for the first one.

Suppose that

$$
£(x)=G^{*} x+\sum_{\ell=1} L_{\ell}^{*} x L_{\ell}+x G
$$

where the operators $G, L_{\ell}$ satisfy the hypothesis AA. Then $£(\mathbb{1})=0$ i.e.

$$
G+G^{*}=-\sum_{\ell=1}^{\infty} L_{\ell}^{*} L_{\ell} .
$$

Letting

$$
i H=-\frac{1}{2} \sum_{\ell=1}^{\infty} L_{\ell}^{*} L_{\ell}-G
$$

the operator $H$ turns out to be symmetric since (formally)

$$
\begin{aligned}
-i H^{*} & =-\frac{1}{2} \sum_{\ell=1}^{\infty} L_{\ell}^{*} L_{\ell^{\prime}}-G^{*} \\
& =-\frac{1}{2} \sum_{\ell=1}^{\infty} L_{\ell}^{*} L_{\ell}+\sum_{\ell=1}^{\infty} L_{\ell}^{*} L_{\ell}+G \\
& =-i H .
\end{aligned}
$$

Therefore we can write $G$ in the form

$$
G=-\frac{1}{2} \sum_{\ell=1}^{\infty} L_{\ell}^{*} L_{\ell}-i H .
$$

Then the form $£$ can be written as

$$
\mathcal{E}(x)=i[H, x]-\frac{1}{2} \sum_{\ell=1}^{\infty}\left(L_{\ell}^{*} L_{\ell} x-2 L_{\ell}^{*} x L_{\ell}+x L_{\ell}^{*} L_{\ell}\right) .
$$

A formal computation yields

$$
\langle v,(£(x y)-x £(y)-£(x) y) u\rangle=\sum_{\ell=1}^{\infty}\left\langle\left[L_{\ell}, x^{*}\right] v,\left[L_{\ell}, y\right] u\right\rangle .
$$


The following is our recipe: given the classical infinitesimal generator then we try to find operators $L_{\ell}$ such that

$$
\langle v,(A(f g)-f A(g)-A(g) f) u\rangle=\sum_{\ell=1}^{\infty}\left\langle\left[L_{\ell}, M(\bar{f})\right] v,\left[L_{\ell}, M(g)\right] u\right\rangle .
$$

Then we try to find a symmetric operator $H$ such that

$$
i[H, M(f)]=£(M(f))+\frac{1}{2} \sum_{\ell=1}^{\infty}\left(L_{\ell}^{*} L_{\ell} M(f)-2 L_{\ell}^{*} M(f) L_{\ell}+M(f) L_{\ell}^{*} L_{\ell}\right)
$$

and consider as operator $G$ an operator defined formally by the right-hand side of (4.4).

Domain problems will be considered later.

\subsection{Diffusion processes on $\mathbb{R}^{d}$}

In this section we will show how to construct a quantum Markov semigroup extending the semigroup of homogeneous diffusions $(y(t))_{t \geq 0}$ on $\mathbb{R}^{d}$ satisfying the stochastic differential equation

$$
d y_{j}(t)=\sum_{k=1}^{d} \sigma_{j k}(y(t)) d w_{k}(t)+b_{j}(y(t)) d t, \quad 1 \leq j \leq d
$$

(where $\left(w_{k}\right)_{1 \leq k \leq n}$ is a $d$ dimensional Wiener process) under the following regularity condition on the matrix $\sigma$ (a square root of the covariance matrix) and on the drift vector $b$ :

\section{Hypothesis D}

1. The functions $\sigma_{j k}: \mathbb{R}^{d} \rightarrow \mathbb{R}(1 \leq j, k \leq d)$ are bounded and four times differentiable with bounded partial derivatives of the first four orders,

2. the functions $b_{j}: \mathbb{R}^{d} \rightarrow \mathbb{R}(1 \leq j \leq d)$ are bounded and three times differentiable with bounded partial derivatives of the first three orders.

It is well known that, if $\sigma$ and $b$ are Lipschitz, then, for every fixed initial condition $y(0)=y_{0}$, there exists a unique Markov process $y$ on $\mathbb{R}^{d}$ satisfying (4.7). We refer to the books [83] by D.W. Stroock and S.R.S. Varadhan, [33] by S.N. Ethier and T.G. Kurtz, [59] by K. Itô, H.P. McKean for detailed results on these processes. Here we use the above stronger assumption simplifying the analytical problems with the unbounded infinitesimal generator.

Let $a=\sigma \sigma^{*}$ and let $\partial_{j}$ denote the partial derivative with respect to the $j$-th coordinate. The infinitesimal generator is the operator

$$
A f=\frac{1}{2} \sum_{j, k=1}^{d} a_{j k} \partial_{j} \partial_{k} f+\sum_{j=1}^{d} b_{j} \partial_{j} f
$$


characterized by Corollary A.2.

Fix $h=L^{2}\left(\mathbb{R}^{d} ; \mathbb{C}\right)$. We compute now formally the operators $G, L_{\ell}$.

For every $f, g \in C_{l}^{2}\left(\mathbb{R}^{d} ; \mathbb{C}\right)$ we have

$$
\begin{aligned}
A(f g)-f A(g)-A(f) g & =\sum_{j, k=1}^{d} a_{j k}\left(\partial_{j} f\right)\left(\partial_{k} g\right) \\
& =\sum_{\ell=1}^{d}\left(\sum_{j=1}^{d} \sigma_{\ell j}\left(\partial_{j} f\right)\right)\left(\sum_{k=1}^{d} \sigma_{\ell k}\left(\partial_{k} g\right)\right)
\end{aligned}
$$

Formula (4.5) suggests to find operators $L_{\ell}$ such that

$$
\sum_{\ell=1}^{\infty}\left[M(f), L_{\ell}^{*}\right] \cdot\left[L_{\ell}, M(g)\right]
$$

coincides with the multiplication operator by the right-hand side of (4.9). This is the case if we consider the operators

$$
L_{\ell}=\sum_{k=1}^{d} \sigma_{\ell k} \partial_{k}+\rho_{\ell}
$$

where $\left(\rho_{\ell}\right)_{1 \leq \ell \leq d}$ is a $d$-dimensional vector with entries in $C^{2}(\mathbb{R} ; \mathbb{R})$ and $L_{\ell}=0$ for $\ell \geq d$. Indeed the commutators $\left[L_{\ell}, M(g)\right],\left[M(f), L_{\ell}^{*}\right]$ coincide with the multiplication operators by

$$
\sum_{k=1}^{d} \sigma_{\ell k}\left(\partial_{k} g\right), \quad \sum_{j=1}^{d} \sigma_{\ell j}\left(\partial_{j} f\right) .
$$

With this choice of the operators $L_{\ell}$ a straightforward computation shows that (with the convention of summation over repeated indices and identification of functions with the corresponding multiplication operator)

$$
\begin{aligned}
L_{\ell}^{*} L_{\ell} M(f)-2 L_{\ell}^{*} M(f) L_{\ell}+M(f) L_{\ell}^{*} L_{\ell} \\
=L_{\ell}^{*}\left[L_{\ell}, M(f)\right]-\left[L_{\ell}^{*}, M(f)\right] L_{\ell} \\
=\left(-\partial_{k} \sigma_{\ell k}+\rho_{\ell}\right) \sigma_{\ell j} M\left(\partial_{j} f\right)+M\left(\partial_{j} f\right) \sigma_{\ell j}\left(\sigma_{\ell k} \partial_{k}+\rho_{\ell}\right) \\
=-\sigma_{\ell k} \sigma_{\ell j} M\left(\partial_{k} \partial_{j} f\right)+\left(2\left(\rho_{\ell} \sigma_{\ell j}\right)-\left(\partial_{k} a_{k j}\right)\right) M\left(\partial_{j} f\right) \\
+\left(\sigma_{\ell j} \sigma_{\ell k}-\sigma_{\ell k} \sigma_{\ell j}\right) M\left(\partial_{j} f\right) \partial_{k} \\
=-\sigma_{\ell k} \sigma_{\ell j} M\left(\partial_{k} \partial_{j} f\right)+\left(2\left(\rho_{\ell} \sigma_{\ell j}\right)-\left(\partial_{k} a_{k j}\right)\right) M\left(\partial_{j} f\right)
\end{aligned}
$$

where we have used

$$
\left(\sigma_{\ell j} \sigma_{\ell k}-\sigma_{\ell k} \sigma_{\ell j}\right)=a_{j k}-a_{k j}=0 .
$$

Therefore the right-hand side of (4.6) coincides with the multiplication by

$$
\left(b_{j}-\frac{1}{2}\left(\partial_{k} a_{k j}\right)+\rho_{\ell} \sigma_{\ell j}\right) M\left(\partial_{j} f\right) \text {. }
$$


Consider a $d$-dimensional vector $\left(\eta_{j}\right)_{1 \leq j \leq d}$ with entries in $C_{b}^{1}(\mathbb{R} ; \mathbb{R})$. Letting

$$
H=-\frac{i}{2} \sum_{j=1}^{d}\left(\eta_{j} \partial_{j}+\partial_{j} \eta_{j}\right)
$$

$\mathcal{E}(M(f))$ coincides with the multiplication operator by $A f$ if and only if

$$
\eta_{j}=b_{j}-\frac{1}{2} \sum_{k=1}^{d}\left(\partial_{k} a_{k j}\right)+\sum_{\ell=1}^{d} \rho_{\ell} \sigma_{\ell j} .
$$

Consider the closure of the operator $G$ with domain $C_{c}^{\infty}\left(\mathbb{R}^{d} ; \mathbb{C}\right)$ given by (4.4) where $L_{\ell}, H$ are defined on $C_{c}^{\infty}\left(\mathbb{R}^{d} ; \mathscr{C}\right)$ by (4.10) and (4.11) respectively. Straightforward computations yield

$$
G=\frac{1}{2} a_{k j} \partial_{k} \partial_{j}+\frac{1}{2}\left(\left(\partial_{k} a_{k j}\right)-2 \eta_{j}\right) \partial_{j}+\frac{1}{2}\left(\left(\partial_{k} \sigma_{\ell k} \rho_{\ell}\right)-\left(\partial_{j} \eta_{j}\right)-\rho_{\ell} \rho_{\ell}\right)
$$

and

$$
G^{*}=\frac{1}{2} a_{k j} \partial_{k} \partial_{j}+\frac{1}{2}\left(\left(\partial_{k} a_{k j}\right)+2 \eta_{j}\right) \partial_{j}+\frac{1}{2}\left(\left(\partial_{k} \sigma_{\ell k} \rho_{\ell}\right)+\left(\partial_{j} \eta_{j}\right)-\rho_{\ell} \rho_{\ell}\right)
$$

We shall construct the minimal quantum dynamical semigroup associated with $G$ and $L_{\ell}$. As first step let us check hypothesis AA.

Proposition 4.4 Suppose that hypothesis $\mathrm{D}$ holds and $\rho_{\ell}, \eta_{\ell} \in C_{b}^{3}\left(\mathbb{R}^{d} ; \mathbb{R}\right)$ for $\ell=1, \ldots, d$. Then the operators $G$ and $L_{\ell}(\ell=1, \ldots, d)$ obtained as closure of the operators with domain $C_{c}^{\infty}\left(\mathbb{R}^{d} ; \mathbb{C}\right)$ defined by (4.13), (4.10) satisfy hypothesis AA.

Proof. By virtue of Theorem A.3 in the Appendix, the operator $G$ is the infinitesimal generator of a strongly continuous contraction semigroup in $h$ and $C_{c}^{\infty}\left(\mathbb{R}^{d} ; \mathbb{C}\right)$ is a core for $G$. Moreover, for every $u, v \in C_{c}^{\infty}\left(\mathbb{R}^{d} ; \mathbb{C}^{j}\right)$, we have

$$
\langle v, G u\rangle+\sum_{\ell=1}^{\infty}\left\langle L_{\ell} v, L_{\ell} u\right\rangle+\langle G v, u\rangle=0
$$

For every $u \in D(G)$, let $\left(u_{n}\right)_{n \geq 1}$ be a sequence of elements of $C_{c}^{\infty}\left(\mathbb{R}^{d} ; \mathbb{C}\right)$ such that

$$
\lim _{n \rightarrow \infty} u_{n}=u, \quad \lim _{n \rightarrow \infty} G u_{n}=G u .
$$

The above identity yields

$$
\sum_{\ell=1}^{d}\left\|L_{\ell}\left(u_{n}-u_{m}\right)\right\|^{2}=-2 \Re e\left\langle\left(u_{n}-u_{m}\right), G\left(u_{n}-u_{m}\right)\right\rangle .
$$


Therefore the sequence $\left(L_{\ell} u_{n}\right)_{n \geq 1}$ also converges to $L_{\ell} u$ for $\ell=1, \ldots, d$ and

$$
\langle u, G u\rangle+\sum_{\ell=1}^{\infty}\left\langle L_{\ell} u, L_{\ell} u\right\rangle+\langle G u, u\rangle=0 .
$$

The identity (3.28) follows then by complex polarisation.

The operators $G, L_{\ell}$, allow to represent the infinitesimal generator (4.8) of the homogeneous classical diffusion (4.7) in Lindablad form.

Proposition 4.5 Suppose that hypothesis $\mathrm{D}$ holds and let $G, L_{\ell}(\ell=1, \ldots, d)$ be as in Proposition 4.4. For every $f \in D(A)$ and every $v, u \in D(G)$ we have

$$
\langle v,(A f) u\rangle=\langle v, f G u\rangle+\sum_{\ell=1}^{\infty}\left\langle L_{\ell} v, f L_{\ell} u\right\rangle+\langle G v, f u\rangle .
$$

Proof. Indeed the above identity holds for $v, u \in C_{c}^{\infty}\left(\mathbb{R}^{d} ; \mathbb{C}\right)$ and $f \in C_{c}^{\infty}\left(\mathbb{R}^{d}\right.$; d) or constant. The conclusion follows easily since these domains are a core for both $G$ and $A$ and the fact (shown in the proof of Proposition 4.4) that convergence of a series $\left(G u_{n}\right)_{n \geq 1}$ with $u \in D(G)$ to $G u$ implies the convergence of $\left(L_{\ell} u_{n}\right)_{n \geq 1}$ to $L u$.

The following is the key step in the construction of the semigroup of a quantum diffusion.

Theorem 4.6 Suppose that hypothesis $\mathrm{D}$ holds and let $\rho_{\ell}, \eta_{\ell} \in C_{b}^{3}\left(\mathbb{R}^{d} ; \mathbb{R}\right)$ for $\ell=1, \ldots, d$. Then the minimal quantum dynamical constructed from $G$ (4.13) and $L_{\ell}(4.10)$ is conservative.

Proof. We shall apply Theorem 3.40. Let $C$ and $\Phi$ be the operators given by

$$
\begin{array}{ll}
D(C)=H^{2}\left(\mathbb{R}^{d} ; \mathscr{C}\right), & C u=c(\sigma, \rho)\left(-\sum_{j=1}^{d} \partial_{j} \partial_{j} u+u\right), \\
D(\Phi)=H^{2}\left(\mathbb{R}^{d} ; \mathscr{C}\right), & \Phi u=-\frac{1}{2} \sum_{\ell=1}^{d} L_{\ell}^{*} L_{\ell} u .
\end{array}
$$

where $c(\sigma, \rho)$ is the constant

$$
c(\sigma, \rho)=2 \sum_{\ell=1}^{d} \max \left\{\left\|\sigma_{\ell 1}\right\|_{\infty}^{2}, \ldots,\left\|\sigma_{\ell d}\right\|_{\infty}^{2},\left\|\rho_{\ell}\right\|_{\infty}^{2}\right\} .
$$

Clearly $C$ and $\Phi$ are self-adjoint by von Neumann's theorem (see [62] Th.3.24 p.275). The linear manifold $C_{c}^{\infty}\left(\mathbb{R}^{d} ; \mathbb{C}\right.$ ) is a core for both (for example by 
Theorem A.3). Moreover it is easy to check that, for every $u \in C_{c}^{\infty}\left(\mathbb{R}^{d} ; \mathbb{C}\right)$, we have

$$
\begin{gathered}
-2 \Re e\langle u, G u\rangle=\sum_{\ell=1}^{\infty}\left\langle L_{\ell} u, L_{\ell} u\right\rangle=\left\langle\Phi^{1 / 2} u, \Phi^{1 / 2} u\right\rangle, \\
\left\langle\Phi^{1 / 2} u, \Phi^{1 / 2} u\right\rangle \leq\left\langle C^{1 / 2} u, C^{1 / 2} u\right\rangle .
\end{gathered}
$$

Since $C_{c}^{\infty}\left(\mathbb{R}^{d} ; \mathbb{C}\right)$ is also core for $G$, the above identities hold for $u \in D(G)$ and $u \in D(C)$. Thus the hypothesis 1 of Theorem 3.40 holds.

In order to apply this result it suffices then to check that the operator $C$ satisfies the hypothesis $\mathbf{C}$.

Notice that, for every $u \in C_{c}^{\infty}\left(\mathbb{R}^{d} ; \mathbb{C}\right)$, integrating by parts we can write the right-hand side of (3.37) in the form

$$
\begin{aligned}
2 \Re e\langle & \langle C u, G u\rangle+\sum_{\ell=1}^{\infty}\left\langle L_{\ell} u, C L_{\ell} u\right\rangle \\
& =i(\langle H u, C u\rangle-\langle C u, H u\rangle) \\
& -\frac{1}{2} \sum_{\ell=1}^{d}\left(\left\langle u, L_{\ell}^{*} L_{\ell} C u\right\rangle-2\left\langle u, L_{\ell}^{*} C L_{\ell} u\right\rangle+\left\langle u, C L_{\ell}^{*} L_{\ell} u\right\rangle\right) \\
& =i(\langle H u, C u\rangle-\langle C u, H u\rangle) \\
& +\frac{1}{2} \sum_{\ell=1}^{d}\left(\left\langle L_{\ell} u,\left[C, L_{\ell}\right] u\right\rangle+\left\langle\left[C, L_{\ell}\right] u, L_{\ell} u\right\rangle\right)
\end{aligned}
$$

Now, for every $u \in C_{c}^{\infty}\left(\mathbb{R}^{d} ; \mathbb{C}\right)$, still integrating by parts we have (with the convention of summation over repeated indices)

$$
\begin{aligned}
|\langle H u, C u\rangle-\langle C u, H u\rangle| & \\
= & \frac{1}{2}\left|\left\langle\left(\eta_{j} \partial_{j}+\partial_{j} \eta_{j}\right) u, \partial_{k} \partial_{k} u\right\rangle-\left\langle\partial_{k} \partial_{k} u,\left(\eta_{j} \partial_{j}+\partial_{j} \eta_{j}\right) u\right\rangle\right| \\
= & \frac{1}{2} \mid-\left\langle\left(\eta_{j} \partial_{j}+\partial_{j} \eta_{j}\right) \partial_{k} u, \partial_{k} u\right\rangle-\left\langle\left(\left(\partial_{k} \eta_{j}\right) \partial_{j}+\partial_{j}\left(\partial_{k} \eta_{j}\right)\right) u, \partial_{k} u\right\rangle \\
& +\left\langle\partial_{k} u,\left(\eta_{j} \partial_{j}+\partial_{j} \eta_{j}\right) \partial_{k} u\right\rangle+\left\langle\partial_{k} u,\left(\left(\partial_{k} \eta_{j}\right) \partial_{j}+\partial_{j}\left(\partial_{k} \eta_{j}\right)\right) u\right\rangle \mid
\end{aligned}
$$

Since $\left(\eta_{j} \partial_{j}+\partial_{j} \eta_{j}\right)$ is antisymmetric on $C_{c}^{\infty}\left(\mathbb{R}^{d} ; \mathbb{C}^{\prime}\right)$ the sum of the first and third term in the right-hand side vanishes and we remain with

$$
\begin{aligned}
& 2|\langle H u, C u\rangle-\langle C u, H u\rangle| \\
& \quad=\left|-\left\langle\left(\left(\partial_{k} \eta_{j}\right) \partial_{j}+\partial_{j}\left(\partial_{k} \eta_{j}\right)\right) u, \partial_{k} u\right\rangle+\left\langle\partial_{k} u,\left(\left(\partial_{k} \eta_{j}\right) \partial_{j}+\partial_{j}\left(\partial_{k} \eta_{j}\right)\right) u\right\rangle\right| \\
& =\left|-2\left\langle\partial_{j} u,\left(\partial_{k} \eta_{j}\right) \partial_{k} u\right\rangle+2\left\langle\partial_{k} u,\left(\partial_{k} \eta_{j}\right) \partial_{j} u\right\rangle+2 \Re e\left\langle u,\left(\partial_{j} \partial_{k} \eta_{j}\right) \partial_{k} u\right\rangle\right|
\end{aligned}
$$

Elementary inequalities lead us to the estimates

$$
2|\langle H u, C u\rangle-\langle C u, H u\rangle| \leq 2 \sup _{k, j}\left\|\partial_{j} \partial_{k} \eta_{j}\right\|_{\infty} \sum_{k=1}^{d}\|u\| \cdot\left\|\partial_{k} u\right\|
$$




$$
\begin{aligned}
& +\quad 4 \sup _{k, j}\left\|\partial_{k} \eta_{j}\right\|_{\infty} \sum_{j, k=1}^{d}\left\|\partial_{j} u\right\| \cdot\left\|\partial_{k} u\right\| \\
& \leq \quad \sup _{k, j}\left\|\partial_{j} \partial_{k} \eta_{j}\right\|_{\infty} \sum_{k=1}^{d}\left(\|u\|^{2}+\left\|\partial_{k} u\right\|^{2}\right) \\
& +\quad 4 \sup _{k, j}\left\|\partial_{k} \eta_{j}\right\|_{\infty}\left(\sum_{k=1}^{d}\left\|\partial_{k} u\right\|\right)^{2} \\
& \leq \quad \sup _{k, j}\left\|\partial_{j} \partial_{k} \eta_{j}\right\|_{\infty} \sum_{k=1}^{d}\left(\|u\|^{2}+\left\|\partial_{k} u\right\|^{2}\right) \\
& +\quad 4 d \sup _{k, j}\left\|\partial_{k} \eta_{j}\right\|_{\infty} \sum_{k=1}^{d}\left\|\partial_{k} u\right\|^{2} .
\end{aligned}
$$

Since

$$
\langle u, C u\rangle=\|u\|^{2}+\sum_{k=1}^{d}\left\|\partial_{k} u\right\|^{2}
$$

for every $u \in C_{c}^{\infty}\left(\mathbb{R}^{d} ; \mathbb{C}^{\prime}\right)$ we have then

$$
|\langle H u, C u\rangle-\langle C u, H u\rangle| \leq b_{1}\langle u, C u\rangle
$$

where

$$
b_{1}=d\left(\sup _{k, j}\left\|\partial_{j} \partial_{k} \eta_{j}\right\|_{\infty}+2 \sup _{k, j}\left\|\partial_{k} \eta_{j}\right\|_{\infty}\right) .
$$

Similar estimates can be done to show that, for every second order differential operator $X$ with coefficients in $C_{b}^{2}\left(\mathbb{R}^{d} ; \mathbb{C}\right)$ and every $u \in C_{c}^{\infty}\left(\mathbb{R}^{d} ; \mathbb{C}\right)$ we have

$$
|\langle u, X u\rangle| \leq b_{2}\langle u, C u\rangle
$$

where $b_{2}$ is a constant depending only on the coefficients of $X$.

Let us estimate now

$$
\sum_{\ell=1}^{d}\left(\left\langle L_{\ell} u,\left[C, L_{\ell}\right] u\right\rangle+\left\langle\left[C, L_{\ell}\right] u, L_{\ell} u\right\rangle\right)
$$

The coefficients of the operators $L_{\ell}(4.10)$ and $L_{\ell}^{*}$ belong to $C_{b}^{3}\left(\mathbb{R}^{d} ; \mathbb{R}\right)$. Therefore, computing explicitely the action of commutators $\left[C, L_{\ell}\right]$ on $u \in C_{c}^{\infty}\left(\mathbb{R}^{d} ; \mathbb{C}\right)$, we can write the above sum as

$$
\begin{aligned}
& -\sum_{\ell, j, m, k}\left(\left\langle\sigma_{\ell m} \partial_{m} u, \partial_{k}\left(\partial_{k} \sigma_{\ell j}\right) \partial_{j} u\right\rangle+\left\langle\partial_{k}\left(\partial_{k} \sigma_{\ell j}\right) \partial_{j} u, \sigma_{\ell m} \partial_{m} u\right\rangle\right) \\
& -2 \Re e \sum_{\ell, j, m}\left\langle\partial_{j}\left(\partial_{j} \rho_{\ell}\right) u, \sigma_{\ell m} \partial_{m} u\right\rangle-2 \Re e \sum_{\ell, j, k}\left\langle\rho_{\ell} u, \partial_{k}\left(\partial_{k} \sigma_{\ell j}\right) \partial_{j} u\right\rangle .
\end{aligned}
$$


The regularity assumption on $\sigma, \rho$ allows us to establish an estimate like (4.15) of the last two sums.

Integrating by parts we can write the first sum as

$$
\begin{aligned}
& -\sum_{\ell, j, m, k}\left\langle\sigma_{\ell m} \partial_{m} u,\left(\partial_{k} \sigma_{\ell j}\right) \partial_{k} \partial_{j} u\right\rangle-\sum_{\ell, j, m, k}\left\langle\sigma_{\ell m} \partial_{m} u,\left(\partial_{k}^{2} \sigma_{\ell m}\right) \partial_{m} u,\right\rangle \\
& +\sum_{\ell, j, m, k}\left\langle\left(\partial_{k} \sigma_{\ell j}\right) \partial_{j} u, \sigma_{\ell m} \partial_{k} \partial_{m} u\right\rangle+\sum_{\ell, j, m, k}\left\langle\left(\partial_{k} \sigma_{\ell j}\right) \partial_{j} u,\left(\partial_{k} \sigma_{\ell m}\right) \partial_{m} u\right\rangle .
\end{aligned}
$$

The second and fourth term satisfy an estimate like (4.15) since they involve only second order partial derivatives. Exchanging $j$ and $m$ in the first sum we can write the sum of the first and third term as

$$
\sum_{\ell, j, m, k}\left(\left\langle\partial_{j} u,\left(\partial_{k} \sigma_{\ell j}\right) \sigma_{\ell m} \partial_{k} \partial_{m} u\right\rangle-\left\langle\partial_{j} u, \sigma_{\ell m}\left(\partial_{k} \sigma_{\ell j}\right) \partial_{k} \partial_{m} u\right\rangle\right)=0
$$

This shows that (4.16) can be estimated by $b_{3}\langle u, C u\rangle$ where $b_{3}$ is a constant depending only on $\sigma$ and $\rho$.

Summing up we have shown that there exists a positive constant $b$ depending only on $\sigma, \rho$ and $\eta$ such that, for every $u \in C_{c}^{\infty}\left(\mathbb{R}^{d} ; \mathbb{C}\right)$, we have

$$
2 \mathfrak{i} e\langle C u, G u\rangle+\sum_{\ell=1}^{\infty}\left\langle L_{\ell} u, C L_{\ell} u\right\rangle \leq b\langle u, C u\rangle .
$$

The above inequality obviously holds for every $u \in C_{-\gamma}^{2}\left(\mathbb{R}^{d} ; \mathbb{C}\right)$ with $\gamma>d / 2$ by a standard approximation argument with mollifyiers.

The domain $C_{-\gamma}^{2}\left(\mathbb{R}^{d} ; \mathscr{C}^{\prime}\right)(\gamma>d / 2)$ is invariant under the semigroup $P(t)$ generated by $G$ and under the resolvents $R(\lambda ; G)$ by Theorem A.1. Therefore it satisfies all the assumptions on the domain $D$ in the hypothesis $\mathrm{C}$. Thus we take $D=C_{-\gamma}^{2}\left(\mathbb{R}^{d} ; \mathbb{C}^{\prime}\right)(\gamma>d / 2)$ The inequality (3.37) for $u \in R(\lambda ; G)(D)$ now follows from $(4.17)$ because, if $u$ belongs to $R(\lambda ; G)(D)$, then by the identity $G R(\lambda ; G)=\lambda R(\lambda ; G)-\mathbb{1}, G u$ belongs also to the domain of $C^{1 / 2}$.

This completes the proof.

By Theorem 4.3 we proved then the following

Proposition 4.7 Suppose that hypothesis $\mathrm{D}$ holds and let $\rho_{\ell}, \eta_{\ell} \in C_{b}^{3}(\mathbb{R} ; \mathbb{R})$ for $\ell=1, \ldots, d$. Then the minimal quantum Markov semigroup constructed from the above operators $G$ and $L_{\ell}$ is an extension to $\mathcal{B}(h)$ of the semigroup of a classical d dimensional diffusion.

When dealing with diffusions on domains with boundaries difficult analytical problems arise to carry on the same program. We refer to the papers [18], [28] and [42] for discussions of some important examples. 


\subsection{A quantum master equation}

In this section we construct a quantum Markov semigroup arising as the solution to a class of master equations in Quantum Optics (see e.g. [15], [47]).

We start by introducing the Heisenberg representation of the $d$-dimensional canonical commutation relations.

Let $h$ be the tensor product Hilbert space of $d$ copies of $l^{2}(N)$ and denote by $\left(e_{\alpha}\right)_{\alpha \in \mathbb{N}^{d}}$ the canonical othonormal basis. Here $\alpha$ is a multindex and the vector $e_{\alpha}$ is the tensor product of unit vectors $e_{\alpha(1)}, \ldots, e_{\alpha(d)}$ taken from the canonical orthonormal basis of each copy of $l^{2}(I N)$ (see the example (1.2)).

We denote by $a_{k}, a_{k}^{*}, N_{k}$ the standard ampliation to $h$ of the annihilation, creation and number operator acting on the $k$-th copy of $l^{2}(I N)$.

The master equation we shall study is the following

$$
\frac{d}{d t} \rho(t)=\mathcal{L}_{*}(\rho(t))
$$

where $\mathcal{L}_{*}$ is the predual operator on density matrices of the Lindblad operator on observables

$$
\begin{aligned}
\mathcal{L}(x)=-\frac{1}{2} \sum_{k=1}^{d} & \left(\mu_{k}\left(a_{k}^{*} a_{k} x-2 a_{k}^{*} x a_{k}+x a_{k}^{*} a_{k}\right)+\lambda_{k}\left(a_{k} a_{k}^{*} x-2 a_{k} x a_{k}^{*}+x a_{k} a_{k}^{*}\right)\right. \\
& \left.+\zeta_{k}\left(a_{k}^{* 2} x-2 a_{k}^{*} x a_{k}^{*}+x a_{k}^{* 2}\right)+\bar{\zeta}_{k}\left(a_{k}^{2} x-2 a_{k} x a_{k}+x a_{k}^{2}\right)\right) \\
& +i \sum_{k=1}^{d}\left[\omega_{k} a_{k}^{*} a_{k}+\xi_{k} a_{k}^{* 2}+\bar{\xi}_{k} a_{k}^{2}+\eta_{k} a_{k}^{*}+\bar{\eta}_{k} a_{k}, x\right]
\end{aligned}
$$

Here $\omega_{k}$ is a real number, $\xi_{k}, \zeta_{k}, \eta_{k}$ are complex numbers, $\mu_{k} \geq 0, \lambda_{k} \geq 0$ and

$$
\left|\zeta_{k}\right|^{2} \leq \mu_{k} \lambda_{k}
$$

for all $k \in\{1, \ldots, d\}$. These inequalitics are necessary and sufficient conditions for $\mathcal{L}$ to be formally (i.e. algebraically) conditional completely positive as it will be clear latcr.

Solving the above quantum master equation means showing that the operator $\mathcal{L}$, interpreted in the form sense as in Chapter 3, can be vicwed as the infinitesimal generator of a quantum Markov semigrup.

As a first step we identify the operators $G, L_{\ell}$ allowing to represent in Lindblad form the above operator $\mathcal{L}$ (denoted $£$ as in Sect. 1 to make clear that it should be understood in the form sense). We proceed as in Sect.1 noting that, for suitable $x, y \in \mathcal{B}(h)$,

$$
£(x y)-x \notin(y)-£(x) y=\sum_{\ell}\left[x, L_{\ell}\right]\left[y, L_{\ell}\right]
$$

must be positive. If $£$ is given by (4.18) and $x=y^{*}$, then a straightforward computation shows that

$$
\sum_{k=1}^{d}\left(\mu_{k}\left[y, a_{k}\right]^{*}\left[y, a_{k}\right]+\zeta_{k}\left[y, a_{k}\right]^{*}\left[y, a_{k}^{*}\right]+\bar{\zeta}_{k}\left[y, a_{k}^{*}\right]^{*}\left[y, a_{k}\right]+\lambda_{k}\left[y, a_{k}^{*}\right]^{*}\left[y, a_{k}^{*}\right]\right)
$$


is positive. Taking operators $y$ obtained by standard ampliation of operators acting on the $k$-th copy of $l^{2}(N)$ we can see that this is the case if and only if each term of the above sum must be positive.

Thus we concentrate on $k=1$ and drop the index to reduce the clutter of the notation. Letting $L=z a+w a^{*}$ with $z, w$ complex numbers to be determined we have

$[y, L]^{*}[y, L]=|z|^{2}[y, a]^{*}[y, a]+\bar{z} w[y, a]^{*}\left[y, a^{*}\right]+z \bar{w}\left[y, a^{*}\right]^{*}[y, a]+|w|^{2}\left[y, a^{*}\right]^{*}\left[y, a^{*}\right]$.

Hence, choosing two numbers $\mu^{\prime}, \lambda^{\prime}$ such that $0 \leq \mu^{\prime} \leq \mu, 0 \leq \lambda^{\prime} \leq \lambda$ and $\mu^{\prime} \lambda^{\prime}=|\zeta|^{2}$, we can find a pair $z, w$ of complex numbers (unique up to a phase factor) such that

$$
|z|^{2}=\mu^{\prime}, \bar{z} w=\zeta, z \bar{w}=\bar{\zeta},|w|^{2}=\lambda^{\prime} .
$$

Therefore can write the decomposition

$$
\begin{aligned}
& \mu[y, a]^{*}[y, a]+\zeta[y, a]^{*}\left[y, a^{*}\right]+\bar{\zeta}\left[y, a^{*}\right]^{*}[y, a]+\lambda\left[y, a^{*}\right]^{*}\left[y, a^{*}\right] \\
& \quad=[y, L]^{*}[y, L]+\left(\mu-\mu^{\prime}\right)[y, a]^{*}[y, a]+\left(\lambda-\lambda^{\prime}\right)\left[y, a^{*}\right]^{*}\left[y, a^{*}\right] .
\end{aligned}
$$

To reduce the number of operators $L_{\ell}$ in the representation in Lindblad form (to the dimension of the minimal representation space associated with the completely positive part of $\mathcal{L}$ we shall choose $\lambda^{\prime}=\lambda$ if $\lambda<\mu$ and $\mu^{\prime}=\mu$, if $\lambda \geq \mu$.

Summing up, in order to represent $\mathcal{L}$ in the Lindblad form, we define (domains will be made precise later), for $k=1, \ldots, d$,

$$
\begin{aligned}
L_{2 k-1} & =z_{k} a_{k}+w_{k} a_{k}^{*}, \\
L_{2 k} & =\left(\mu_{k}-\mu_{k}^{\prime}\right)^{1 / 2} a_{k}, \quad \text { if } \mu_{k}>\lambda_{k}, \\
L_{2 k} & =\left(\lambda_{k}-\lambda_{k}^{\prime}\right)^{1 / 2} a_{k}^{*}, \quad \text { if } \mu_{k} \leq \lambda_{k} .
\end{aligned}
$$

With this choice of $L_{\ell}$, a natural choice for the operator $G$ is the following

$$
G=-\frac{1}{2} \sum_{\ell=1}^{2 d} L_{\ell}^{*} L_{\ell}-i H
$$

where

$$
H=\sum_{k=1}^{d}\left(\omega_{k} a_{k}^{*} a_{k}+\xi_{k} a_{k}^{* 2}+\bar{\xi}_{k} a_{k}^{2}+\eta_{k} a_{k}^{*}+\bar{\eta}_{k} a_{k}\right) .
$$

The above operators are obviously defined on the linear manifold $D$ spanned by the elements $e_{\alpha}$ of the canonical orthonormal basis of $h$. Now we shall construct rigorously the quantum Markov semigroup associated with an appropriated extension of these operators.

As a first step we shall prove that the closure of the above operator $G$ with domain $D$ generates a strongly continuous contraction semigroup on $h$ applying the following result due to Palle E.T. Jorgensen (see [73] Th.2 p.398) 
Theorem 4.8 Let $G$ be a dissipative linear operator on a Hilbert space $h$. Let $\left(D_{n}\right)_{n \geq 1}$ be an increasing family of closed subspaces of $h$ whose union is dense in $h$ and contained in the domain of $G$ and let $P_{D_{n}}$, be the orthogonal projection of h onto $D_{n}$. Suppose that there exists an integer $n_{0}$ such that $G D_{n} \subseteq D_{n+n_{0}}$ for all $n \geq 1$. Then the closure $\bar{G}$ generates a strongly continuous contraction semigroup on $h$ and $\cup_{n \geq 1} D_{n}$ is a core for $\bar{G}$, if there exists a sequence $\left(c_{n}\right)_{n \geq 1}$ in $\mathbb{R}_{+}$such that $\left\|G P_{D_{n}}-P_{D_{n}} G P_{D_{n}}\right\| \leq c_{n}$ for all $n$ and

$$
\sum_{n=1}^{\infty} c_{n}^{-1}=\infty \text {. }
$$

With the above notation let $D_{n}$ be the linear manifold spanned by vectors 6 with $|\alpha| \leq n$. Clearly $D=\cup_{n \geq 1} D_{n}$. The opcrator $G$ is obviously densely defined and dissipative. Therefore it is closable (see e.g. [21] Lemma 3.1.14 p.175) and its closure, denoted $\bar{G}$ is dissipative.

Proposition 4.9 The operator $\bar{G}$ is the infinitesimal generator of a strongly continuous contraction semigroup on $h$.

Proof. Clearly, by the explicit form of the action of creation and annihilation operators on vectors $e_{\alpha}$, the operator $G$ maps $D_{n}$ into $D_{n+2}$ for all $n \geq 0$. In order to apply Theorem 4.8 we shall show that the norm $\left\|G P_{D_{n}}-P_{D_{n}} G P_{D_{n}}\right\|$ grows at most linearly. To this end let us fix $u=\sum_{|\alpha|<n} z_{\alpha} e_{\alpha}$ a vector in $D_{n}$. A straightforward computation yields

$$
\begin{aligned}
& \left(G P_{D_{n}}-P_{D_{n}} G P_{D_{n}}\right) u=-i \sum_{k=1}^{d} \eta_{k} \sum_{|\alpha|=n}(\alpha(k)+1)^{1 / 2} z_{\alpha} e_{\alpha+1_{k}} \\
& \quad-\sum_{k=1}^{d}\left(\frac{\zeta_{k}}{2}+\xi_{k}\right) \sum_{|\alpha|=n-1}((\alpha(k)+1)(\alpha(k)+2))^{1 / 2} z_{\alpha} e_{\alpha+2_{k}} \\
& \quad-\sum_{k=1}^{d}\left(\frac{\zeta_{k}}{2}+\xi_{k}\right) \sum_{|\alpha|=n}((\alpha(k)+1)(\alpha(k)+2))^{1 / 2} z_{\alpha} e_{\alpha+2_{k}} .
\end{aligned}
$$

The squared norm of the third term can written

$$
\begin{aligned}
& \sum_{k, k^{\prime}=1}^{d} \sum_{|\alpha|,\left|\alpha^{\prime}\right|=n}\left(\frac{\bar{\zeta}_{k^{\prime}}}{2}+\bar{\xi}_{k^{\prime}}\right)\left(\frac{\zeta_{k}}{2}+\xi_{k}\right) \\
& \cdot\left(\left(\alpha^{\prime}\left(k^{\prime}\right)+1\right)\left(\alpha^{\prime}\left(k^{\prime}\right)+2\right)(\alpha(k)+1)(\alpha(k)+2)\right)^{1 / 2} \bar{z}_{\alpha^{\prime}} z_{\alpha}\left\langle e_{\alpha^{\prime}+2_{k^{\prime}}}, e_{\alpha+2 z_{k}}\right\rangle
\end{aligned}
$$

Note that, for each $k, k^{\prime} \in\{1, \ldots, d\}$ and $\alpha \in \mathbb{N}^{d}$ with $|\alpha|=n$, there exists exactly a multindex $\alpha^{\prime}=\beta\left(\alpha, k, k^{\prime}\right)$ such that the scalar product $\left\langle e_{\alpha^{\prime}+2_{k^{\prime}}}, e_{\alpha+2 k}\right\rangle$ does not vanish. Therefore the squared norm of the third term can be estimated 
by

$$
\begin{aligned}
(n+2)^{2} & \sum_{k, k^{\prime}=1}^{d} \sum_{|\alpha|=n}\left|\frac{\bar{\zeta}_{k^{\prime}}}{2}+\bar{\xi}_{k^{\prime}}\right|\left|\frac{\zeta_{k}}{2}+\xi_{k}\right|\left|\bar{z}_{\beta\left(\alpha, k, k^{\prime}\right)}\right|\left|z_{\alpha}\right| \\
& \leq \frac{(n+2)^{2}}{2} \sum_{k, k^{\prime}=1}^{d}\left|\frac{\bar{\zeta}_{k^{\prime}}}{2}+\bar{\xi}_{k^{\prime}}\right|\left|\frac{\zeta_{k}}{2}+\xi_{k}\right| \sum_{|\alpha|=n}\left(\left|\bar{z}_{\beta\left(\alpha, k, k^{\prime}\right)}\right|^{2}+\left|z_{\alpha}\right|^{2}\right) \\
& =(n+2)^{2}\|u\|^{2}\left(\sum_{k=1}^{d}\left|\frac{\zeta_{k}}{2}+\xi_{k}\right|\right)^{2} .
\end{aligned}
$$

In a similar way we can majorize the squared norms of the first and second term respectively by

$$
(n+1)^{2}\|u\|^{2}\left(\sum_{k=1}^{d}\left|\eta_{k}\right|\right)^{2}, \quad(n+2)^{2}\|u\|^{2}\left(\sum_{k=1}^{d}\left|\frac{\zeta_{k}}{2}+\xi_{k}\right|\right)^{2} .
$$

By the elementary $|r+s+t|^{2} \leq 3\left(|r|^{2}+|s|^{2}+|t|^{2}\right)$ we have then

$$
\left\|G P_{D_{n}}-P_{D_{n}} G P_{D_{n}} u\right\| \leq \sqrt{3}\left(2\left(\sum_{k=1}^{d}\left|\frac{\zeta_{k}}{2}+\xi_{k}\right|\right)^{2}+\left(\sum_{k=1}^{d}\left|\eta_{k}\right|\right)^{2}\right)^{1 / 2}(n+2)\|u\| .
$$

Since the series $\sum_{n}(n+2)^{-1}$ is divergent the proof is complete.

A similar argument (or an application of an extension of Nelson's theorem on analytic vectors, [79] Th.X.40 p.206) allows us to prove the following

Proposition 4.10 The closure $\Phi$ of the operator on $h$ with domain $D$ defined by

$$
\sum_{\ell=1}^{2 d} L_{\ell}^{*} L_{\ell}=\sum_{k=1}^{d}\left(\mu_{k} a_{k}^{*} a_{k}+\zeta_{k} a_{k}^{* 2}+\bar{\zeta}_{k} a_{k}^{2}+\lambda_{k} a_{k} a_{k}^{*}\right)
$$

is essentially self-adjoint.

Let us denote by $G$ both the operator $G$ and its closure. The operators $L_{\ell}$ can be extended to the domain of $G$ and further extended to the domain

$$
\left\{\left.u \in h\left|u=\sum_{\alpha} z_{\alpha} e_{\alpha}, \sum_{\alpha}\right| \alpha\right|^{1 / 2}\left|z_{\alpha}\right|^{2}<\infty\right\}
$$

by standard arguments. Moreover, it follows readily that the hypothesis AA holds.

In order to solve the quantum master equation (4.18) it suffices now to prove that the minimal quantum dynamical semigroup associated with the operators $G, L_{\ell}(\ell=1, \ldots, 2 d)$ is Markov. To this end we shall choose a suitable operator 
$C$ and apply the results of Section 3. The choice of the operator $C$, however, depends upon some non-degeneracy conditions on the parameters $\mu_{k}, \lambda_{k}, \zeta_{k}$, $\omega_{k}, \xi_{k}, \eta_{k}$. We shall study only the following case and make some comments on the solution of our problem in the other cases at the end.

Hypothesis ND - For each $k \in\{1, \ldots, d\}$ at least one among $\mu_{k}, \lambda_{k},\left|\omega_{k}\right|$, $\left|\xi_{k}\right|$ is nonzero.

Proposition 4.11 Suppose that the hypothesis ND holds. Let $C$ be the operator defined by

$D(C)=\left\{\left.u \in h\left|u=\sum_{\alpha} z_{\alpha} e_{\alpha}, \sum_{\alpha}\left(|\alpha|^{2}+1\right)\right| z_{\alpha}\right|^{2}<\infty\right\}, \quad C u=c \sum_{\alpha}|\alpha| z_{\alpha} e_{\alpha}$.

where $c>0$ is a constant. Then $C$ is self-adjoint and $D$ is a core for $C$. Moreover:

(1) the domain of $G$ coincides with the domain of $C$,

(2) if $c>\max _{1 \leq k \leq d}\left(\mu_{k}+\lambda_{k}+2\left|\zeta_{k}\right|\right)$, for all $u \in D$ we have

$$
\langle u, \Phi u\rangle \leq\langle u, C u\rangle \text {. }
$$

Proof. For every $\alpha \in \mathbb{N}^{d}$ and every $n \geq 1$ we have $C^{n} e_{\alpha}=|\alpha|^{n} e_{\alpha}$. Therefore $C$ is self-adjoint and $D$ is a core for $C$ by Nelson's theorem on analytic vectors (see e.g. [79] Th.X.39 p.202). (This can be shown alternatively applying [73] Corollary 1 p.397 with $D_{n}$ as before).

A direct computation allows to show that for each $u=\sum_{\alpha} z_{\alpha} e_{\alpha}$ in the domain of $C$ the sequence $\left(u_{n}\right)_{n \geq 1}$ defined by $u_{n}=\sum_{|\alpha| \leq n} z_{\alpha} e_{\alpha}$ for $n \geq 1$ converges strongly to $u$ and $\left(G u_{n}\right)_{n \geq 1}$ converges strongly to in $h$. Since $G$ is closed, it follows that $u$ belongs to the domain of $G$. Thus the domain of $G$ is contained in the domain of $C$.

On the other hand the restriction of $G$ to $D$ is relatively bounded with respect to $C$. Indeed, for all $u \in D$ and all $k \in\{1, \ldots, d\}$ we have

$$
\left\|a_{k}^{* 2} u\right\|^{2}=\left\langle u, a_{k}^{2} a_{k}^{* 2} u\right\rangle=\left\langle a_{k} a_{k}^{*} u,\left(a_{k} a_{k}^{*}+\mathbb{1}\right) u\right\rangle \leq\left\|\left(a_{k} a_{k}^{*}+\mathbb{1}\right) u\right\|^{2} .
$$

So that

$$
\left\|a_{k}^{* 2} u\right\| \leq\|C u\|+\|u\| .
$$

Moreover, similar estimates can be easily obtained for the other terms in the sum defining $G$. Thus $G$ is relatively bounded with respect to $C$.

It follows that the domain of $G$ contains that of $C$ and (1) follows.

Finally, in order to prove (2) it suffices to note that, for all $u \in D$ and all $k \in\{1, \ldots, d\}$, we have

$$
\begin{aligned}
\left\langle u,\left(\zeta_{k} a_{k}^{* 2}+\bar{\zeta}_{k} a_{k}^{2}\right) u\right\rangle & =2 \Re e \zeta_{k}\left\langle u, a_{k}^{* 2} u\right\rangle \\
& \leq 2\left|\zeta_{k}\right| \cdot\left\|a_{k} u\right\| \cdot\left\|a_{k}^{*} u\right\| \\
& \leq 2\left|\zeta_{k}\right|\left(\left\langle u, a_{k}^{*} a_{k} u\right\rangle \cdot\left\langle u, a_{k} a_{k}^{*} u\right\rangle\right)^{1 / 2} \\
& \leq 2\left|\zeta_{k}\right|\left\langle u, a_{k} a_{k}^{*} u\right\rangle .
\end{aligned}
$$


Then (2) follows easily.

Proposition 4.12 Suppose that the hypothesis ND holds. Then the minimal quantum dynamical semigroup associated with the operators $G, L_{\ell}(\ell=$ $1, \ldots, 2 d)$ is Markov.

Proof. We apply Corollary (3.41). The domains of $G$ and $C$ coincide by the above Proposition. We check now the hypotheses a), b), c) of the Corollary.

The hypothesis a) clearly holds because $D$ is invariant uncter the $L_{\ell}$ 's.

The hypothesis b) also holds by Proposition 4.11 if we choose $c$ big enough.

Finally we check condition c). Since $D$ is an invariant domain for the operators $G, L_{\ell}, C$ it suffices to compute algebraically $£(C)$. Indeed the left-hand side of (3.41) can be written in the form $\langle u, £(C) u\rangle$. Although there are at times a product of eight operators in the working below it should be remembered that these expressions should strictly be interpreted as forms, and so everything is well-defined by the invariance of $D$ under the action of creation and annihilation operators.

Note that, for every $k, m \in\{1, \ldots, d\}$, by the canonical commutation relations $\left[a_{k}, a_{m}^{*}\right]=\delta_{k, m} \mathbb{1}$ (on the domain $D$ ), we have

$$
\begin{aligned}
a_{k}^{*} a_{k}\left(a_{m} a_{m}^{*}\right)-2 a_{k}^{*}\left(a_{m} a_{m}^{*}\right) a_{k}+\left(a_{m} a_{m}^{*}\right) a_{k}^{*} a_{k} & =-2 \delta_{k, m}, \\
a_{k} a_{k}^{*}\left(a_{m} a_{m}^{*}\right)-2 a_{k}\left(a_{m} a_{m}^{*}\right) a_{k}^{*}+\left(a_{m} a_{m}^{*}\right) a_{k} a_{k}^{*} & =2 \delta_{k, m}, \\
a_{k}^{* 2}\left(a_{m} a_{m}^{*}\right)-2 a_{k}^{*}\left(a_{m} a_{m}^{*}\right) a_{k}^{*}+\left(a_{m} a_{m}^{*}\right) a_{k}^{* 2} & =0, \\
a_{k}^{2}\left(a_{m} a_{m}^{*}\right)-2 a_{k}\left(a_{m} a_{m}^{*}\right) a_{k}+\left(a_{m} a_{m}^{*}\right) a_{k}^{2} & =0, \\
{\left[\omega_{k} a_{k}^{*} a_{k}+\xi_{k} a_{k}^{* 2}+\bar{\xi}_{k} a_{k}^{2}+\eta_{k} a_{k}^{*}+\bar{\eta}_{k} a_{k}, a_{m} a_{m}^{*}\right] } & \\
=\delta_{k, m}\left(2 \overline{\xi_{k}} a_{k}^{2}-2 \xi_{k} a_{k}^{* 2}+\bar{\eta}_{k} a_{k}-\eta_{k} a_{k}^{*}\right) &
\end{aligned}
$$

Therefore, for $u \in D$, we obtain

$$
\langle u, \mathcal{E}(C) u\rangle=\sum_{k=1}^{d}\left\langle u,\left(2 \bar{\xi}_{k} a_{k}^{2}-2 \xi_{k} a_{k}^{* 2}+\bar{\eta}_{k} a_{k}-\eta_{k} a_{k}^{*}\right) u\right\rangle+2 \sum_{k=1}^{d}\left(\lambda_{k}-\mu_{k}\right)\|u\|^{2} .
$$

The same arguments of the proof of Proposition 4.11 lead to the inequality

$$
\left\langle u,\left(\bar{\xi}_{k} a_{k}^{2}-\xi_{k} a_{k}^{* 2}\right) u\right\rangle \leq 2\left|\xi_{k}\right|\left\langle u, a_{k} a_{k}^{*} u\right\rangle,
$$

and

$$
\left\langle u,\left(\bar{\eta}_{k} a_{k}-\eta_{k} a_{k}^{*}\right) u\right\rangle=2\left|\eta_{k}\right| \cdot\|u\| \cdot\left\|a_{k} u\right\| \leq\left|\eta_{k}\right|\left(\|u\|^{2}+\left\|a_{k} u\right\|^{2}\right)=\left|\eta_{k}\right|\left\langle u, a_{k} a_{k}^{*} u\right\rangle .
$$

Summing up we find

$$
\begin{aligned}
\langle u, £(C) u\rangle & \leq c^{-1} \max _{1 \leq k \leq d}\left\{4\left|\xi_{k}\right|+\left|\eta_{k}\right|\right\}\langle u, C u\rangle+2 \sum_{k=1}^{d}\left(\lambda_{k}-\mu_{k}\right)\|u\|^{2} \\
& \leq c^{-1} \max \left\{\max _{1 \leq k \leq d}\left\{4\left|\xi_{k}\right|+\left|\eta_{k}\right|\right\}, 2 \sum_{k=1}^{d}\left(\lambda_{k}-\mu_{k}\right)\right\}\langle u, C u\rangle .
\end{aligned}
$$


This shows that condition c) of Corollary (3.41) holds. Then the proof is complete.

Remark. If the hypothesis ND does not hold let

$$
\begin{aligned}
& K_{1}=\left\{k \in\{1, \ldots, d\}\left|\mu_{k}+\lambda_{k}+\right| \omega_{k}|+| \xi_{k} \mid>0\right\}, \\
& K_{2}=\left\{k \in\{1, \ldots, d\}-K_{1}|| \eta_{k} \mid>0\right\} .
\end{aligned}
$$

Then we can show that the minimal quantum dynamical semigroup associated with the operators $G, L_{\ell}(\ell=1, \ldots, 2 d)$ is Markov by the same arguments with the choice of the operator $C$ given, up to a multiplicative constant $c$, by

$$
\sum_{k \in K_{1}} a_{k} a_{k}^{*}+\sum_{k \in K_{2}}\left(a_{k} a_{k}^{*}\right)^{1 / 2}
$$

